\title{
Topical steroid therapy in atopic dermatitis in theory and practice
}

\author{
Renata Jeziorkowska ${ }^{1}$ Anna Sysa-Jędrzejowska², Zbigniew Samochocki ${ }^{1}$
}

${ }^{1}$ Department of Dermatology, Medical University of Warsaw, Warsaw, Poland

Head of the Department: Prof. Lidia Rudnicka MD, PhD

${ }^{2}$ Department of Dermatology, University of Social Sciences, Lodz, Poland

Head of the Department: Prof. Franciszek Seneczko MD, PhD

\begin{abstract}
Introduction: Topical glucocorticosteroids (GCSs) are commonly used in treatment of atopic dermatitis (AD).

Aim: To assess the patients' compliance with the recommended instructions of the therapy.

Material and methods: The study involved 141 adult AD patients. The clinical course of AD and its treatment with GCSs during the last year were analysed.

Results: In the periods of exacerbation the lesions involved $10-50 \%$ of the skin surface area. Outpatient treatment in specialised dermatological and/or allergology clinics was given to $93 \%$ of the study subjects. Sixty-five out of 141 patients regularly attended medical control examinations. Glucocorticosteroids, mostly very potent ones (70.2\%), were applied to all the subjects. $66.7 \%$ of patients obtained no information about their medications' anti-inflammatory potential. The substances were applied more frequently than twice daily by $36.4 \%$ of the patients. Seventy-two of 141 subjects applied GCSs both temporarily and in the long-term treatment, for 8.3 weeks on average. In the long-term treatment, in which very potent GCSs predominated (70.7\%), no one used intermittent therapy. One hundred and thirty patients introduced their own modifications to the instructions concerning GCSs use, among which $37.7 \%$ changed the site of application, $58.5 \%$ prolonged the duration of application and $49.5 \%$ shortened it or occasionally temporarily withdrew the prescribed drug. None of the patients knew the fingertip unit method of dose assessment. Apart from steroid therapy, 56.7\% of the patients carried out regular care treatment. Conclusions: The AD patients need to be thoroughly educated by the medical staff in the topical GCSs therapy in atopic dermatitis.
\end{abstract}

Key words: atopic dermatitis, glucocorticosteroids, topical treatment.

\section{Introduction}

Glucocorticosteroid hormones (GCSs) of the adrenal cortex constitute the main group of drugs in modern medicine. A common feature of this group of drugs is their structure consisting of 17 carbon atoms bonded in four rings and one side chain. Modifications of individual compounds include changes within the ring structures as well as in the side chain. Glucocorticosteroids were introduced to dermatological treatment in 1952 in the form of hydrocortisone. Owing to their lipophilicity, they penetrate the cell membrane easily and bind with their specific receptor in the cytoplasm. The transcrip- tion of mRNA becomes inhibited or stimulated, leading to a multidirectional activity causing a lower expression of adhesion molecules, limitation of T-cell migration, decline in the synthesis of proinflammatory cytokines and stimulation of lipocortins synthesis [1, 2]. Transdermal absorption of GCSs depends on their chemical structure, excipient used, degree of epidermal barrier damage, application area and region of the skin and patient's age. The penetration of ointments is the deepest, followed then by creams, lotions, gels and aerosols. The absorption in children is higher because of poorly developed cornified, granular and spinous layers. Thin epidermis and loose structure of the links connecting it to the dermis in the

Address for correspondence: Prof. Zbigniew Samochocki MD, PhD, Department of Dermatology, Medical University of Warsaw, 82a Koszykowa St, 02-008 Warsaw, Poland, phone: +48 604325 665, e-mail: samochocki@gmail.com Received: 23.12.2013, accepted: 2.01.2014. 
crotch, eyelids, face and skin folds are the causes of increased penetration of the substances deep into the skin. Topical GCS application can cause the development of significant side effects, such as skin atrophy, vascular damage, steroid-induced acne, contact hypersensitivity or systemic action [3]. Safe topical steroid therapy is based on three fundamental principles: correctly selected potency of action, the dose, and the method of application [4]. Therefore, the importance of patients' education by the doctor is commonly stressed.

In view of their antiproliferative, anti-inflammatory and immunosuppressive effects, GCSs find wide use in the topical treatment of various skin diseases, including allergic ones. Atopic dermatitis (AD) is a chronic inflammatory dermatosis of complex, not fully elucidated aetiopathogenesis, resulting in absence of causal treatment. Topical therapeutic treatment is established individually, taking into account the type and intensity of skin lesions and patient's age. Since those patients express dysfunction of the congenital and acquired immunological system of the skin [5, 6], the aim of the treatment is to inhibit the topical inflammatory process. Glucocorticosteroids are the first-choice drugs to treat skin lesions in AD [4].

In view of xeroderma developed in the course of the disease, which is associated with a various degree of epidermal barrier damage [7], care procedures play an important role in the therapeutic process. Emollients are recommended for permanent use, particularly as a complementary treatment to intermittent therapy with GCSs, and they are the basic element of therapeutic conduct both in the period of exacerbations and remissions of skin lesions [4].

\section{Aim}

The aim of the study was to assess the knowledge of correct topical corticosteroid therapy principles and adherence to them by the patients with AD.

\section{Material and methods}

The analysed data were obtained from the history and medical documentation of 141 patients (56 wom- en and 85 men) aged 18-70 years old, average age: 29.7 years old, treated for AD in the Department Outpatient Clinic and hospitalised in the Department of Dermatology, Warsaw Medical University. The patients met the Hanifin and Rajka diagnostic criteria [8]. The duration of the disease was from 2 to 20 years, average duration: 11.3 years. The clinical course of $A D$ and its treatment during the last year were assessed. The potency of action of the topically applied glucocorticosteroids was determined according to the four-grade European classification [9]. The application at least once a day for a period not exceeding 14 days and repeated not more than four times a year was regarded as temporary use of GCSs. In the remaining cases GCS treatment was qualified as long-term therapy.

The extent of skin lesions during exacerbations was determined by the rule of nines [10].

The permission for the study conduct was issued by the local Bioethical Committee.

\section{Results}

The study group was mainly treated in dermatological centres (91/140, 64.7\%) and allergology centres (40/141, 28.4\%). Only $7.1 \%$ of the patients (10/141) were under exclusive care of the GP physician. Sixty-five out of 141 patients (46.1\%) regularly visited their attending doctors while 76/141 patients (53.9\%) came for irregular visits to random doctors.

During exacerbation periods the skin lesions involved from $10 \%$ to $50 \%$ of the skin surface area, $30 \%$ on average. In 97/141 subjects (68.8\%), the lesions were always located on the face and neck.

All study subjects applied GCSs. One hundred twenty-nine patients (91.5\%) declared that they were informed about the principles of topical treatment and the type of GCSs used by their attending doctor.

The knowledge of the potency of action of very strong and strong GCSs was declared by $33.3 \%$ and $32.7 \%$ of the patients, respectively. This percentage for drugs of moderate and low potency of action was $52 \%$ and $80 \%$, respectively.

Table 1 presents the frequency and method of application for individual GCS types.

Table 1. Frequency and method of application of individual types of GCS in 141 patients with AD

\begin{tabular}{|c|c|c|c|c|c|c|c|c|}
\hline \multirow[t]{3}{*}{ Type of GCSs } & \multirow{2}{*}{\multicolumn{2}{|c|}{$\begin{array}{l}\text { Number of patients } \\
\qquad N=141\end{array}$}} & \multicolumn{6}{|c|}{ Mode of application } \\
\hline & & & \multicolumn{2}{|c|}{$>2 \times$ daily } & \multicolumn{2}{|c|}{ Temporary } & \multicolumn{2}{|c|}{ Long-term } \\
\hline & $n$ & $\%$ & $n^{*}$ & $\%$ & $n^{*}$ & $\%$ & $n^{*}$ & $\%$ \\
\hline Very high potency of action & 99 & 70.2 & 36 & 36.4 & 29 & 29.3 & 70 & 70.7 \\
\hline High potency of action & 98 & 69.5 & 35 & 35.7 & 30 & 30.6 & 68 & 69.4 \\
\hline Moderate potency of action & 50 & 35.5 & 25 & 50.0 & 24 & 48.0 & 26 & 52.0 \\
\hline Low potency of action & 110 & 78.0 & 56 & 50.9 & 50 & 49.5 & 60 & 54.5 \\
\hline
\end{tabular}

${ }^{*} 100 \%=$ number of patients using individual types of GCSs. 
Glucocorticosteroids of very high (clobetasol propionate, betamethasone dipropionate - ointments), high (mometasone furoate, fluticasone propionate, methylprednisolone aceponate, betamethasone valerate, hydrocortisone butyrate - ointments) and low (flumethasone pivalate solution, hydrocortisone acetate $1 \%$ - cream) potency of action were used in all the study group in similar percentages $-70.2,69.5$ and 78.8 , respectively. This percentage for substances of moderate potency of action (flucinolone acetonide - ointment, fluticasone propionate-cream) was 35.5. The medications from the four mentioned groups were applied for more than twice a day by $36.4 \%, 35.7 \%$, $50.9 \%$ and $50.0 \%$ of the patients, respectively.

Glucocorticosteroids were temporarily used by 69/141 patients (48.9\%), while $72 / 141$ patients $(51.2 \%)$ used them both temporarily and as the long-term treatment, for 8.3 weeks on average.

In the long-term treatment mainly GCSs of very high (70.7\%) and high (69.4\%) potency of action were applied. These percentages for steroids of moderate and low potency of action were 52.0 and 54.5 , respectively.

None of the patients receiving the long-term treatment used intermittent therapy.

In the study group, 130/141 (92.2\%) patients changed the instructions concerning GCSs application on their own, among which 49/130 (37.7\%) changed the site of application, 76/130 (58.5\%) prolonged and 64/130 (49.2\%) shortened the duration of application or even temporarily abandoned the prescribed drug.

None of the patients knew the acceptable monthly dose or its determination by the fingertip unit method.

Apart from the GCS therapy, 80/141 patients (56.7\%) performed their regular care procedures (baths, emollients, greasing).

\section{Discussion}

Regular care provided by a "permanent" specialist plays a significant role in achieving therapeutic success in chronic diseases. Although $93 \%$ of the study patients received specialist care, only $46 \%$ were regularly consulted by doctors. This percentage is low taking into account the fact that the group consisted of patients with severe course of the disease, which was evidenced by the high extensiveness of the lesions during recurrences (involvement of $30 \%$ of the skin area on average). Therefore, it is not surprising that GCSs were topically applied in all the cases.

Although the majority of patients (91.5\%) declared obtaining information about the type of applied drugs from the attending doctors, the knowledge of their potency was varied and incomplete. Most frequent correct answers (80\%) concerned substances of very low potency of action. In the case of medications of higher-than-moderate potency of action this percentage did not exceed $34 \%$. A similar finding was reported by other authors, both among adult patients with $A D[11,12]$ and guardians of ill children [13]. This relationship could be explained by the fact that hydrocortisone is a very popular drug, available over the counter and advertised in mass media. Therefore, the knowledge about this compound was the best.

The basic principle of topical therapy with GCSs according to Ring [14] is their application in time "for as short as possible and as long as it is needed". In the topical treatment of $A D$, the substances of high potency of action are only applied during exacerbation periods once a day for not more than 14 days. With increasing improvement they should be changed for less potent drugs. In the long-term treatment (over 14 days) the medications of higher-than-moderate potency of action are not recommended. Initially they should be applied twice a day, and then, with increasing improvement, once a day. Further dose reduction includes application every second day, then twice a week alternately with neutral excipients. This results from the fact that GCSs remain in the horny layer after treatment discontinuation for a long time since this layer is a kind of a peculiar reservoir for this group of drugs. Moreover, the replacement of strongly acting substances to weak ones and then gradual reduction of application frequency prevents from "rebound phenomenon", which provides intensification of skin lesions after abrupt drug withdrawal.

In the study group, GCSs of low as well as high and very high potency of action were used in the similar percentage of cases. The significant percentage of strong-acting medications in the study group could be explained by high intensity of their skin lesions. High frequency of recommended strong-acting drugs usage was not dependent on regularity of medical visits and the care provided by a regular or a random doctor (data not presented in the results). Such expected relationship may be explained by the fact that the emergency visits to random doctors concerned mainly patients during the periods of significant exacerbations. This may result from the fact that the dose reduction (replacement of strong GCSs with weak ones) and recommendation of prescribing only weak GCSs in chronic cases are not performed.

In the long-term therapy, in which no patient used the intermittent method, very strong and strong steroids predominated. The observed therapeutic management is not in agreement with the recommended standards of AD topical treatment since it constitutes an additional cause of epidermal barrier dysfunction. It was demonstrated that very strong GCSs damage the epidermal barrier structure and function not only in the long-term treatment [15] but also after a short-lasting application [16]. Moreover, daily multiple application of a GCS can also lead to a blockade of cytoplasmatic receptors for these drugs and can cause paradoxical lack of response to the treatment [3]. The potential risk of side effects developed after the topical steroid treatment in the study 
group was increased by the fact that in most patients the skin lesions were located on the face and involved over $1 / 3$ of the body surface area, on average, and the average duration of the long-term application was 2 months. Although only $10 \%$ of the patients declared that they were not informed about the principles of their therapy by the doctors, the significant majority of subjects introduced their own modifications to the instructions - mainly prolonged the duration of applications and changed the application site. The presumption is that this lack of compliance was another significant factor of the observed incorrect therapeutic procedure. It could also result from the fact that in the study group the recurrences involved large skin areas and the majority of patients irregularly sought medical advice. Therefore, they prolonged the duration of strong GCSs application on their own expecting a more rapid regression of the inflammatory lesions. In the study of Polish AD patients' population Jenerowicz et al. [11] demonstrated that the patients used mainly GCSS of a low and moderate potency of action. The authors, however, failed to describe the extent of the lesions during recurrences and to present the division into temporary and the long-term applications.

The lack of adequate knowledge in the field of potency of action of individual groups of medications and resulting possibilities of potential side effects seems to be the cause of GCSs application incompatible with the standards of topical therapy in atopic eczema connected with prolonged usage of high anti-inflammatory potential drugs. On the other hand, incorrect assessment of the potency of GCSs action may start unjustified fear of the treatment and can be a possible cause of shortening the duration of application or even abandoning it. The phenomenon of so called "steroid phobia" was reported in $50-70 \%$ of $A D$ patients $[11,17,18]$, although not all the patients abandoned the prescribed treatment for this particular reason. In the performed study, the duration of prescribed treatment was shortened or even temporarily abandoned, in spite of the recommendations, by 64/141 (45.4\%) patients. Interestingly enough, the symptoms of steroid phobia were observed only in 10\% of patients with asthma and the greatest fear of GCSs therapy was found in patients who had never used that group of drugs [14].

It can be predicted that described abnormalities of the therapy reported by the patients in the medical history in the last year, were present also in the previous years. Taking into account the long duration of the disease (average duration 11 years), it significantly increases the risk of developing corticosteroid-induced side effects.

According to the consensus proposed by the Dermatology Working Group [12], prior to treatment with topical GCSs, patients should obtain information concerning: a) the maximal weekly FTU (fingertip unit) dose; b) the time in which the prescribed packages should be used; c) the principles of increasing or decreasing the potency of applied medications; d) approximate duration of the treatment and indications for its repetition; e) realistic goals of the therapy; f) treatment efficacy assessment time frame; g) potential adverse effects: what kind of symptoms and signs in particular attention should be paid to, when the treatment should be discontinued, when the doctor should be visited; h) particular caution during pregnancy or breast feeding. It seems that such clear information will significantly facilitate topical GCS therapy and significantly reduce the number of mistakes in its conduct.

In the case of oral drugs administration, the dose prescribed by the doctor and taken by the patient is strictly controlled. For the drugs applied externally the dose is usually neither determined by the doctor nor known by the patient. During the long-term treatment, the monthly dose of topical GCSs should not exceed $15 \mathrm{~g}$ in babies, $30 \mathrm{~g}$ in children, $60 \mathrm{~g}$ in adolescents and $90 \mathrm{~g}$ in adults [4]. In 1991, Long and Finaly [19] published a simple method of dose determination for topically applied GCSs, the so-called "fingertip unit" (FTU). It is assumed that 1 FTU stands for an ointment portion of $5 \mathrm{~mm}$ diameter placed on the distal phalanx of the index finger, which corresponds to $500 \mathrm{mg}$. It is the amount of ointment or cream which makes it possible to cover the area of pathological lesions equal to that of two palms of an adult person. It was calculated that in adults, the foot, including its dorsum, the hand, the anterior surface of the trunk, including the abdomen, the back and the buttocks, the face and the neck, the upper limb, including the hand, and the lower limb including the foot require doses equal to 2, 1 , 7, 7, 2.5, 4 and 8 FTUs, respectively. It was even suggested to include FTU description in manufacturers' leaflets enclosed to the drugs [12]. The authors' observations show that the doctors do not recognize the necessity of assessing the total dose and the described simple method of its determination finds no practical use.

In our own studies, it was also demonstrated that a significant percentage (43.3\%) of AD patients did not perform any care procedures, which evidenced that this part of the therapeutic management is very frequently neglected. It may be caused by lack of knowledge about practical importance of baths (they increase hydration of the horny layer and facilitate penetration of administered drugs, they remove irritating/allergizing factors) and topical application of emollients (they regenerate the epidermal barrier; it is possible to reduce the dose by increasing the penetration of GCSs and they prevent the development of steroid-induced side effects) [20, 21]. A high price of medications for atopic skin care may be another factor limiting their use. The care procedures require high regularity and application twice a day, which is frequently very difficult because of the patients' lifestyle. Moreover, in the presented study, the majority of patients were males who accept the care procedures worse than women. 
The conducted study confirms the necessity of organized education for AD patients. The most important factors are the motivation to the treatment and clear transfer of information concerning the therapy. The forms of such extended education vary among the countries [22] and its usefulness is confirmed by numerous observations. German studies demonstrated that a 12 -hour educational programme involving 433 outpatients with AD caused a statistically significant reduction in the SCORAD value and an increase in the quality of life index by compliance to the principles of the administered treatment over the year. These results were compared to $377 \mathrm{pa}-$ tients in whom that form of education was not used, yet the therapeutic conditions of both groups were similar [23]. Another study also demonstrated the usefulness of 12-hour education in a significant reduction of fear of topical GCSs application [24].

\section{Conclusions}

In spite of the obtained guidelines from the attending doctors, the lack of compliance with the basic principles of steroid therapy demonstrates that the patients need to be thoroughly educated by the medical staff in the field of topical GCSs therapy in atopic dermatitis.

\section{Conflict of interest}

The authors declare no conflict of interest.

\section{References}

1. Ahluwalia A. Topical glucocorticoids and the skin mechanisms of action an update. Mediators Inflamm 1998; 7 : 183-93.

2. Drake LA, Dinehart SM, Farmer ER et al. Guidelines of care for the use of topical glucocorticosteroids. Acta Dermatol 1996; 35: 615-9.

3. Hengge UR, Ruzicka T, Schwartz RA, et al. Adverse effects of topical glucocorticoids. J Am Acad Dermatol 2006; 54: 1-15.

4. Darsow U, Wollenberg A, Simon D, et al. ETFAD/EADV eczema task force 2009 position paper on diagnosis and treatment of atopic dermatitis. JEADV 2010; 24: 317-28.

5. Werfel T. The role of leukocytes, keratinocytes, and allergen-specific IgE in the development of atopic dermatitis. J Invest Dermatol 2009; 129: 1878-91.

6. De Benedetto A, Agnihothri R, McGirt L, et al. Atopic dermatitis: a caused by innate immune defects? I Invest Dermatol 2009; 129: 14-30.

7. Cork M, Danby S, Vasilopoulos Y, et al. Epidermal barrier dysfunction in atopic dermatitis. J Invest Dermatol 2009; 129: 1892-908.

8. Hanifin JM, Rajka G. Diagnostic features of atopic dermatitis. Acta Dermatol Venerol (Stockh.) 1980; Suppl. 92: 44-7.

9. Katsambas AC, Lotti TM (eds). European handbook of dermatological treatments. Springer, Berlin 2003.

10. Wallance AB. The exposure treatment of burns. Lancet 1951; 2: 501-3.

11. Jenerowicz D, Czarnecka-Operacz M, Silny W. Corticosteroid phobia in patients with atopic dermatitis. Wiad Lek 2005; 58: 607-15.
12. Bewley A. Dermatology Working Group. Expert consensus: time of change the way we advise our patients to use topical corticosteroids. Br J Dermatol 2008; 158: 917-20.

13. Beattite PE, Lewis-Jones MS. Parental knowledge of topical therapies in the treatment of childhood atopic dermatitis. Clin Exp Dermatol 2003; 28: 549-53.

14. Kercher M, Williams S, Lehmann P. Topical treatment with glucocorticoids. In: Handbook of atopic dermatitis. Ring J, Przybilla B, Ruzicka T (eds.) Springer-Verlag, Berlin 2006; 471-91.

15. Sheu HM, Lee JY, Chai CY, Kuo KW. Depletiation of stratum corneum intercellular lipid lamelle and barrier function abnormalities after long-term topical corticosteroids. Br J Dermatol 1997; 136: 884-90.

16. Kao J, Man M, Fowler A, et al. Short-term glucocorticoid treatment compromises both permeability barrier homeostasis and stratum corneum integrity: inhibition of epidermal lipid synthesis accounts for functional abnormalities. J Invest Dermatol 2003; 120: 456-64.

17. Charman CR, Williams HC. The use of corticosteroids and corticosteroid phobia in atopic dermatitis. Clin Dermatol 2003; 21: 193-200.

18. Charman CR, Morris AD, Williams HC. Topical corticosteroid phobia in patients with atopic eczema. Br J Dermatol 2000; 142: 931-6.

19. Long CC, Finaly AY. The finger-tip unit - a new practical measure. Clin Exp Dermatol 1991; 16: 444-7.

20. Hanifin J, Cooper K, Ho V, et al. Guideline of care for atopic dermatitis. J Am Acad Dermatol 2004; 3: 391-404.

21. Grimalt R, Mengeaud V, Combazard F. The steroid-sparing effect of an emollient therapy in infants with atopic dermatitis: a randomized controlled study. Dermatology 2007; 214: 61-7.

22. Stadle JF, Bernier C, Ball A, et al. Therapeutic patient education in atopic dermatitis: worldwide experiences. Pediatr Dermatol 2013; 30: 329-4.

23. Staab, D, Diepgen T, Fartasch M, et al. Age-related, structured education programmes improve the management of atopic dermatitis in children and adolescents: results of the German Atopic Dermatitis Intervention Study (GADIS). BMJ 2006; 332: 933-8.

24. Staab D, Ruden U, Kehrt R, et al. The impact of childhood atopic dermatitis on quality of life of family. Dermatol Psychosom 2000; 1: 173-8. 